\title{
HMFcalc: An Online Tool for Calculating Dark Matter Halo Mass Functions
}

\author{
S. G. Murray ${ }^{1,2}$, C. Power ${ }^{1,2}$ \& A. S. G. Robotham ${ }^{1}$ \\ ${ }^{1}$ ICRAR, University of Western Australia, 35 Stirling Highway, Crawley, Western Australia 6009, Australia \\ 2 ARC Centre of Excellence for All-Sky Astrophysics (CAASTRO)
}

\begin{abstract}
The dark matter halo mass function (HMF) is a characteristic property of cosmological structure formation models, quantifying the number density of dark matter haloes per unit mass in the Universe. A key goal of current and planned large galaxy surveys is to measure the HMF and to use it to test theories of dark matter and dark energy. We present a new web application for calculating the HMF - the frontend HMFcalc and the engine hmf. HMFcalc has been designed to be flexible, efficient and easy to use, providing observational and theoretical astronomers alike with the means to explore standard functional forms of the HMF or to tailor their own. We outline the theoretical background needed to compute the HMF, we show how it has been implemented in hmf, and finally we provide worked examples that illustrate HMFcalc's versatility as an analysis tool.
\end{abstract}

\section{Introduction}

There is now a wealth of compelling observational evidence that we live in a Universe whose matter content is predominantly dark ( 84\%; cf. Ade et al. 2013) and nonbaryonic in nature (cf. Bergström, 2000). Our theories of cosmological structure formation predict that this dark matter clusters into massive gravitationally bound structures called haloes. The dark matter halo mass function (hereafter HMF) quantifies the number of these haloes per unit comoving volume of the Universe as a function of their mass. The HMF is sensitive to the cosmological parameters, primarily the mass-energy density of dark matter $\Omega_{c}$ and dark energy $\Omega_{\Lambda}$ (e.g. Murray et al., 2013), but it $\checkmark$ also depends on the nature of the dark matter. The standard Cold Dark Matter (CDM) model predicts an HMF in which the number of haloes increases with decreasing halo mass $M$ approximately as $M^{-1.8}$ (e.g. Lukić et al. 2007, Bhattacharya et al., 2011), whereas viable Warm Dark Matter (WDM) models predict fewer haloes than $>$ the CDM model at low masses(e.g. Schneider et al., 2013 , Pacucci et al. 2013). The potential of the HMF as a probe of dark matter and dark energy is widely recognised (e.g. Tinker and Kravtsov, 2008; Vikhlinin et al., 2009) and is one of the key science drivers of current and planned future galaxy surveys (Driver, 2011; Pierre et al., 2011).

Cosmological $N$-body simulations are now established as the tool for studying the HMF (cf. the recent review by Knebe et al. 2013), but the information contained in a simulation is usually distilled and recast in a more compact form. Usually this is the comoving number density of haloes per unit logarithm of the halo mass $M$,

$$
\frac{d n}{d \ln M}=M \cdot \frac{\rho_{0}}{M^{2}} f(\sigma)\left|\frac{d \ln \sigma}{d \ln M}\right| ;
$$

here $\sigma$ and $\rho_{0}$ are the cosmology-dependent mass variance and mean density and $f(\sigma)$ represents the functional form that defines a particular HMF fit. Eq 1 is not difficult to compute, but neither is it straightforward. We have developed an easy-to-use and flexible web-application to compute Eq 1. which we call $\mathrm{hmf}$, and its frontend HMFcalc which can be accessed at http://hmf.icrar. org. HMFcalc can be used in a number of ways, including as

- a standard against which to check one's own code;

- an easy-to-use interface to generate HMFs against which to check observational/simulations data; and

- a visually intuitive way to explore the effects of cosmology on the HMF.

The objective of this paper is to present a detailed overview of $\mathrm{hmf}$ and HMFcalc, describing its implementation and the underlying philosophy for this approach, as well as providing some worked examples that illustrate its usefulness and versatility.

The paper is structured as follows. In $₫ 2$ we provide the theoretical background necessary to compute the HMF, setting out a compilation of HMF fitting functions drawn from the literature and demonstrating how the HMF differs in CDM and WDM models. In $\S 3$ we describe our implementation of $\mathrm{hmf}$ and HMFcalc and discuss the algorithms and methods used. In $\$ 4$ we present some worked examples using HMFcalc and finally in $\$ 5$ we summarise our plans for extending HMFcalc's functionality and usability. 


\section{The Halo Mass Function (HMF)}

The HMF quantifies the number of dark matter haloes per unit mass per unit comoving volume of the Universe,

$$
\frac{d n}{d \ln M}=M \cdot \frac{\rho_{0}}{M^{2}} f(\sigma)\left|\frac{d \ln \sigma}{d \ln M}\right|
$$

where $f(\sigma)$ is the fitting function that we shall return to shortly, $\rho_{0}$ is the mean density of the Universe and $\sigma$ is the rms variance of mass within a sphere of radius $R$ that contains mass $M$,

$$
M=\frac{4 \pi \rho_{0}}{3} R^{3} \text {. }
$$

The mass variance is calculated via the integral,

$$
\sigma^{2}(R)=\frac{1}{2 \pi^{2}} \int_{0}^{\infty} k^{2} P(k) W^{2}(k R) d k
$$

where $k=2 \pi / \lambda$ is the wavenumber corresponding to the wavelength $\lambda$ of the fluctuation, $P(k)$ is the linear power spectrum (see below) and $W(k R)$ is the Fourier transform of the top-hat window function ${ }^{1}$ (a sphere with sharp edges in real space), i.e.

$$
W(k R)=\frac{3[\sin (k R)-k R \cos (k R)]}{(k R)^{3}} .
$$

The right-most factor of $\mathrm{Eq} 1$ can be written as

$$
\frac{d \ln \sigma}{d \ln M}=\frac{3}{2 \sigma^{2} \pi^{2} R^{4}} \int_{0}^{\infty} \frac{d W^{2}(k R)}{d M} \frac{P(k)}{k^{2}} d k
$$

using Eq 2 to relate $M$ to $R$, and writing

$$
\begin{aligned}
\frac{d W^{2}}{d M}= & {[\sin (k R)-k R \cos (k R)] \times } \\
& {\left[\sin (k R)\left(1-\frac{3}{(k R)^{2}}\right)+3 \frac{\cos (k R)}{k R}\right] . }
\end{aligned}
$$

The window function and its derivative are functions of the product $k R$, but we evaluate Eqs 3 and 5 by integrating over $k$. For this reason care must be taken when solving the integrals numerically to ensure that the results are converged. We demonstrate why in Fig 1, where we plot $\int_{0}^{k R} W^{2}(x) d x$ and $\int_{k R}^{\infty} W^{2}(x) d x$. The integral $\int_{0}^{k R} W^{2}(x) d x$ allows us to identify an upper limit on the minimum $k R$ required for convergence; we want the range of $k R$ for any $R$ to have a minimum that bounds the non-zero parts of the function. As a rule of thumb, we identify this as the value of $k R$ for which $95 \%$ of $\int_{0}^{\infty} W^{2}(x) d x$ is kept; from

\footnotetext{
${ }^{1}$ Note that there is freedom in the choice of window function; we follow the convention in which the window function is a top-hat in real space, but we could adopt a Gaussian in real space, or a tophat in Fourier space. The dependence of $\sigma$ on the choice of window function is well known and the implications have been explored in studies such as Schneider et al. (2013). We shall return to this point in 95
}

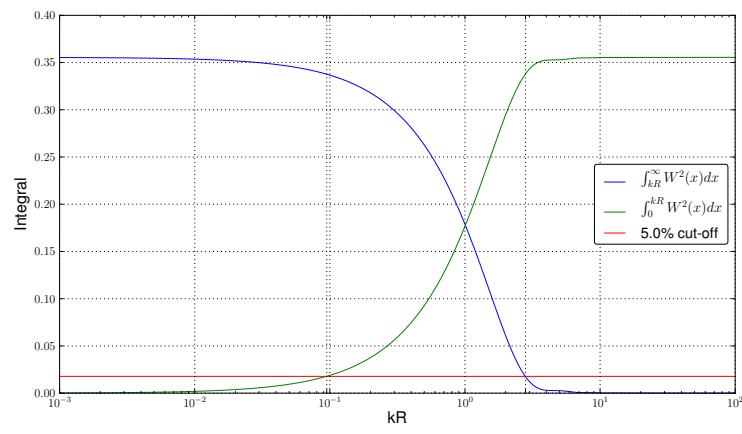

Figure 1: The integral of the window function gives an upper limit of 0.1 on the minimum of the $k R$ range, and a lower limit of 3 on the maximum of the $k R$ range, with a $95 \%$ contribution of the total integral.

Fig 1, this corresponds to $k R=0.1$ and so we have the condition,

$$
k_{\min } R_{\max }<0.1
$$

Repeating this exercise for $\int_{k R}^{\infty} W^{2}(x) d x$, we deduce

$$
k_{\max } R_{\min }>3 .
$$

These bounds on $k R$ are implemented in hmf by default, which issues a warning if they are not obeyed. Usually the limits on $k$ to which the power spectrum is extrapolated far exceed these rules for any sensible mass range, but instances can arise where more stringent limits on $k$ are required (cf. 4.2 ).

The linear power spectrum $P(k)$ characterises the distribution of matter density perturbations as a function of wavenumber $k$; formally it is computed by averaging over $|\delta(\vec{k})|$, the square of the Fourier-transformed real-space density fluctuations $\delta(\vec{x})=\rho(\vec{x}) / \bar{\rho}-1$, where $\vec{x}$ and $\vec{k}$ are the spatial coordinate and wave vector respectively. We express it as

$$
P(k)=A k^{n} T^{2}(k),
$$

where $T(k)$ is the transfer function, $A$ is the normalisation constant and $n$ is the spectral index. We follow convention and use the cosmological parameter $\sigma_{8}$, which measures the mass variance on a scale of $8 h^{-1} \mathrm{Mpc}$, to calculate $A$. The primordial power spectrum, imprinted during the epoch of inflation during the first moments after the Big Bang, is expected to have a form $P(k) \propto k^{n}$. The transfer function quantifies how this primordial form is modified on different scales, and it is particularly sensitive to the nature of the dark matter and the baryon density parameter $\Omega_{\mathrm{b}}$. We use the public Code for Anisotropies in the Microwave Background (CAMB) (Lewis et al., 2000) to compute our transfer functions.

\subsection{Fitting Functions}

As noted in the introduction, it is the fitting function $f(\sigma)$ that defines a particular HMF fit. Early analytical 


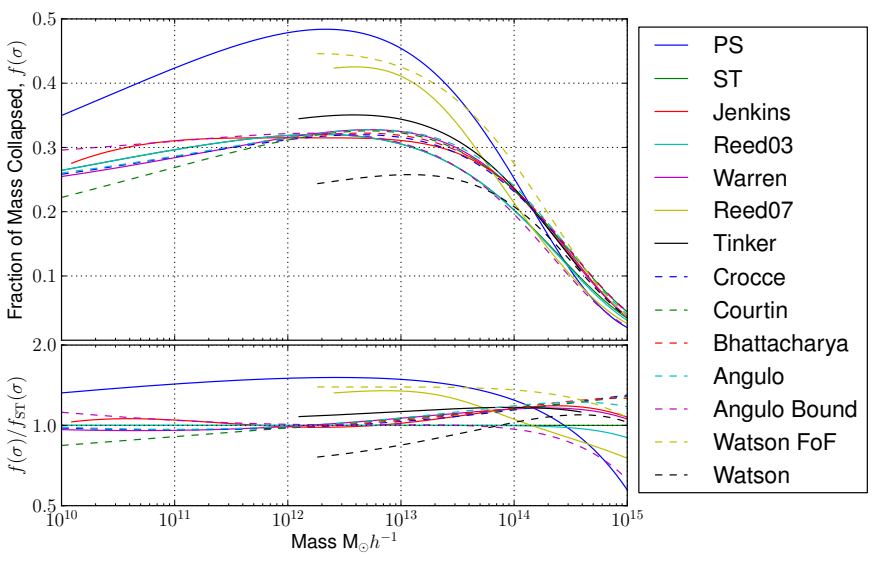

Figure 2: Upper: all fitting functions at redshift zero over a large mass range (limits placed as appropriate on each function). Lower: each fitting function divided by the Sheth-Tormen fit.

work by Press and Schechter (1974) (hereafter PS) and Bond et al. (1991) established a simple form for $f(\sigma)$ by assuming that haloes form by spherical collapse, finding

$$
f(\sigma)=\sqrt{\frac{2}{\pi}} \frac{\delta_{c}}{\sigma} \exp \left(-\frac{\delta_{c}^{2}}{2 \sigma^{2}}\right)
$$

where $\delta_{c} \simeq 1.686$ is the critical overdensity for spherical collapse.

However, $N$-body simulations of cosmological structure formation have revealed that the PS form underestimates the abundance of higher mass haloes and overestimates the abundance of lower mass haloes. (e.g. Sheth et al. 2001; White, 2002, Lukić et al., 2007). Sheth et al. (2001) (hereafter ST) explored an extension to the PS formalism by considering ellipsoidal rather than spherical collapse and obtained a form for the mass function that is identical to $\mathrm{Eq} 1$ but with a modified $f(\sigma)$. Subsequent studies have largely adopted the same philosophical approach of assuming that the HMF can be expressed in the form of Eq 1 and using $f(\sigma)$ to characterise the HMF.

Table 1 provides a concise summary of the forms for $f(\sigma)$ that have appeared in the literature to date and which are included in HMFcalc, and we list also the cosmology and mass and redshift ranges over which the fits have been $\operatorname{mad} \AA^{2}$. Each of these fitting functions are plotted in Fig 2 for comparison.

\subsection{Redshift Dependence}

In general it is assumed that the form of the fitting function $f(\sigma)$ is insensitive to redshift, and so it is through the rms mass variance $\sigma(M, z)$ that redshift dependence is

\footnotetext{
${ }^{2}$ Note the parameters $A, a, b, c$ for the Tinker fit are actually dependent on $\Delta_{\text {vir }}$. See Tinker and Kravtsov (2008) or the code for details.
}

captured. The evolution of $\sigma(M, z)$ is governed by the growth factor,

$$
d(z)=\frac{D^{+}(z)}{D^{+}(z=0)} .
$$

where $D^{+}(a)$ is

$$
D^{+}(z)=\frac{5 \Omega_{m}}{2} \frac{H(z)}{H_{0}} \int_{z}^{\infty} \frac{\left(1+z^{\prime}\right) d z^{\prime}}{\left[H\left(z^{\prime}\right) / H_{0}\right]^{3}},
$$

and $H_{0}$ is the present-day Hubble parameter with

$$
H(z)=H_{0} \sqrt{\Omega_{m}(1+z)^{3}+\left(1-\Omega_{m}\right)}
$$

its value at $z$.

\subsection{Warm Dark Matter Models}

The default in HMFcalc is to calculate the HMF assuming an underlying Cold Dark Matter (CDM) model, but we have included the option to calculate the HMF in Warm Dark Matter (WDM) alternatives. Small-scale power is suppressed in a WDM model compared to its CDM counterpart, reflecting the free-streaming of WDM particles in the early Universe, dispersing small-scale density perturbations that would otherwise collapse via gravitational instability to form low-mass dark matter haloes. The influence of the dark matter model on structure formation is readily apparent in Fig 2.3 , which shows the projected dark matter density at $z=0$ in a $20 h^{-1} \mathrm{Mpc}$ patch in a fiducial CDM model (left panel) and in a WDM model assuming a WDM particle mass of $m_{X}=0.5 \mathrm{keV} / c^{2}$. Although the large-scale features (filaments and voids, positions of the most massive haloes) are the same in both runs, a wealth of small-scale structure - low mass dark matter haloes is evident in the CDM run but not in the WDM run.

The standard approach to capturing the effect of WDM on the power spectrum is to filter the corresponding CDM power spectrum on small scales by an additional transfer function, i.e. $P(k)=P_{C D M} T_{k}^{X^{2}}$. The transfer function of Bode et al. (2001) is widely used, and it is the one we adopt in HMFcalc; here

$$
T_{k}^{X}=\left(1+(\alpha k)^{2 \nu}\right)^{-5 / \nu}
$$

with $\nu=1.2$ and

$$
\alpha=0.048\left(\frac{\Omega_{X}}{0.4}\right)^{.15}\left(\frac{h}{.65}\right)^{1 / 3}\left(\frac{1}{m_{X}}\right)^{1.15}\left(\frac{1.5}{g_{X}}\right)^{.29}
$$

with $\Omega_{X}$ the current fractional density of the WDM particle (this can be taken as equivalent to the CDM density $\Omega_{c d m}$ in a single-species WDM model), $m_{X}$ is the particle mass in $\mathrm{keV}$, and $g_{X}$ controls the abundance of the species relative to photons and has the fiducial value of 1.5 for a light neutrino.

By default in HMFcalc, we assume that $\nu$ and $g_{X}$ are set to their fiducial values and allow only a single-species model; the only free parameter that we allow is $m_{X}$. Fig 2.3 shows a fiducial CDM and WDM HMFs for $m_{X}=0.5$, 
Table 1: Compilation of Fitting Functions

\begin{tabular}{|c|c|c|c|c|}
\hline REF. & Fitting Function $f(\sigma)$ & Mass RANGE & $\begin{array}{l}\text { REDSHIFT } \\
\text { RANGE } \\
\end{array}$ & $\begin{array}{l}\text { COSMOLOGY } \\
\text { FitTED } \\
\end{array}$ \\
\hline \begin{tabular}{|l|} 
Press and \\
Schechter \\
1974 \\
\end{tabular} & $f_{\mathrm{PS}}(\sigma)=\sqrt{\frac{2}{\pi}} \frac{\delta_{c}}{\sigma} \exp \left[-\frac{\delta_{c}^{2}}{2 \sigma^{2}}\right]$ & - & - & - \\
\hline $\begin{array}{l}\text { Sheth et al. } \\
2001\end{array}$ & $\begin{array}{l}f_{\mathrm{ST}}(\sigma)=A \sqrt{\frac{2 a}{\pi}}\left[1+\left(\frac{\sigma^{2}}{a \delta_{c}^{2}}\right)^{p}\right] \frac{\delta_{c}}{\sigma} \exp \left[-\frac{a \delta_{c}^{2}}{2 \sigma^{2}}\right] \\
A=0.3222, a=0.707, p=0.3 .\end{array}$ & - & - & $\begin{array}{l}\text { Einstein-de } \\
\text { Sitter }\end{array}$ \\
\hline \begin{tabular}{|l|} 
Jenkins \\
et al. $(2001)$ \\
\end{tabular} & $f_{\mathrm{J}}(\sigma)=0.315 \exp \left[\left|\ln \sigma^{-1}+0.61\right|^{3.8}\right]$ & $\begin{array}{c}-1.2<\ln \sigma^{-1}< \\
1.05\end{array}$ & $0-5$ & $\tau \mathrm{CDM}, \Lambda \mathrm{CDM}$ \\
\hline Reed et al. & $f_{\mathrm{R} 03}(\sigma)=f_{S T}(\sigma) \exp \left[\frac{-0.7}{\sigma \cosh (2 \sigma)^{5}}\right]$ & $\begin{array}{c}-1.7<\ln \sigma^{-1}< \\
0.9\end{array}$ & $0-15$ & $\begin{array}{l}\Omega_{M}=0.3 \\
\Omega_{\Lambda}=0.7\end{array}$ \\
\hline \begin{tabular}{|l|} 
Warren \\
et al. $(2006)$ \\
\end{tabular} & $f_{\mathrm{W}}(\sigma)=0.7234\left(\sigma^{-1.625}+0.2538\right) \exp \left[\frac{-1.1982}{\sigma^{2}}\right]$ & $\begin{array}{c}10^{10} \mathrm{M}_{\odot}<M< \\
10^{15} \mathrm{M}_{\odot}\end{array}$ & 0 & $\begin{array}{l}\Lambda \mathrm{CDM}: \\
\text { WMAP1 }\end{array}$ \\
\hline$\frac{\text { Reed et al. }}{2007}$ & $\begin{array}{l}f_{\mathrm{R} 07}(\sigma)=\nu \exp \left[-\frac{c a \frac{\delta_{c}^{2}}{\sigma^{2}}}{2}-\frac{0.03\left(\frac{\delta_{c}}{\sigma}\right)^{0.6}}{\left(n_{\mathrm{eff}}+3\right)^{2}}\right] \\
\times A \sqrt{\frac{2 a}{\pi}}\left[1+\left(\frac{1}{\frac{\delta_{c}^{2}}{\sigma^{2} a}}\right)^{p}+0.6 G_{1}(\sigma)+0.4 G_{2}(\sigma)\right] \\
n_{\mathrm{eff}}=6 \frac{d \log \sigma^{-1}}{d \log M}-3, G_{1}(\sigma)=\exp \left[-\frac{\ln \left(\sigma^{-1}-0.4\right)^{2}}{0.72}\right] \\
G_{2}(\sigma)=\exp \left[-\frac{\ln \left(\sigma^{-1}-0.75\right)^{2}}{0.08}\right]\end{array}$ & $\begin{array}{c}-1.7<\ln \sigma^{-1}< \\
0.9\end{array}$ & $0-30$ & $\begin{array}{l}\Lambda \mathrm{CDM}: \\
\text { WMAP1 }\end{array}$ \\
\hline \begin{tabular}{|l|} 
Tinker and \\
Kravtsov \\
2008
\end{tabular} & $\begin{array}{l}f_{\mathrm{T}}(\sigma, z)=A\left(\left(\frac{b}{\sigma}\right)^{a}+1\right) \exp \left[-\frac{c}{\sigma^{2}}\right], \\
A=0.186(1+z)^{-0.14}, a=1.47(1+z)^{-0.06}, \\
b=2.57(1+z)^{-\alpha}, c=1.19, \\
\alpha=\exp \left[-\left(\frac{0.75}{\ln \left(\Delta_{\mathrm{vir}} / 75\right)}\right)^{1.2}\right]\end{array}$ & $\begin{array}{c}-0.6<\ln \sigma^{-1}< \\
0.4\end{array}$ & $0-2.5$ & $\begin{array}{l}\Lambda \mathrm{CDM}: \\
\text { WMAP1, } \\
\text { WMAP3+ }\end{array}$ \\
\hline \begin{tabular}{|l|} 
Crocce \\
et al. 2010, \\
\end{tabular} & $\begin{array}{l}f_{\mathrm{Cr}}(\sigma)=A\left(\sigma^{-a}+b\right) \exp \left[-\frac{c}{\sigma^{2}}\right] \\
A=0.58(1+z)^{-0.13}, a=1.37(1+z)^{-0.15} \\
b=0.3(1+z)^{-0.084}, c=1.036(1+z)^{-0.024}\end{array}$ & $\begin{array}{c}10^{10.5} \mathrm{M}_{\odot}<M< \\
10^{15.5} \mathrm{M}_{\odot}\end{array}$ & $0-2$ & $\begin{array}{l}\left(\Omega_{M}, \Omega_{\Lambda}, n, h, \sigma_{8}\right) \\
=(0.25,0.75 \\
0.95,0.7,0.8) \\
\end{array}$ \\
\hline \begin{tabular}{|l|} 
Courtin \\
et al. $(2010)$
\end{tabular} & $\begin{array}{l}f_{\mathrm{Co}}(\sigma)=f_{\mathrm{ST}}(\sigma) \\
A=0.348, a=0.695, p=0.1\end{array}$ & $\begin{array}{c}-0.8<\ln \sigma^{-1}< \\
0.7\end{array}$ & 0 & $\begin{array}{l}\Lambda \mathrm{CDM}: \\
\text { WMAP5 }\end{array}$ \\
\hline \begin{tabular}{|l|} 
Bhattacharya \\
et al. $(2011)$ \\
\end{tabular} & $\begin{array}{l}f_{\mathrm{B}}(\sigma, z)= \\
A \sqrt{\frac{2}{\pi}} \exp \left[-\frac{a \delta_{c}^{2}}{2 \sigma^{2}}\right]\left[1+\left(a \frac{\delta_{c}^{2}}{\sigma^{2}}\right)^{-p}\right]\left(\frac{\delta_{c}^{2}}{\sigma^{2}} \sqrt{a}\right)^{q} \\
A=0.333(1+z)^{-0.11}, a=0.788(1+z)^{-0.01} \\
p=0.807, q=1.795\end{array}$ & $\begin{array}{c}10^{11.8} \mathrm{M}_{\odot}<M< \\
10^{15.5} \mathrm{M}_{\odot}\end{array}$ & $0-2$ & $w \mathrm{CDM}+$ \\
\hline \begin{tabular}{|l|} 
Angulo \\
et al. $(2012)$ \\
\end{tabular} & $\begin{array}{l}f_{\mathrm{A}}(\sigma)=A\left[\left(\frac{b}{\sigma}\right)^{a}+1\right] \exp \left[-\frac{c}{\sigma^{2}}\right] \\
(A, a, b, c)=(0.201,1.7,2.08,1.172) \text { or } \\
(A, a, b, c)_{\mathrm{SUB}}=(0.265,1.9,1.675,1.4)\end{array}$ & $\begin{array}{c}10^{8} \mathrm{M}_{\odot}<M< \\
10^{16} \mathrm{M}_{\odot}\end{array}$ & 0 & $\begin{array}{l}\Lambda \mathrm{CDM}: \\
\text { WMAP1 }\end{array}$ \\
\hline \begin{tabular}{|l|} 
Watson \\
et al. $(2013)$ \\
\end{tabular} & $\begin{array}{l}f_{\mathrm{W}_{\mathrm{FOF}}}(\sigma, z)=f_{\mathrm{T}}(\sigma, z) \\
A=0.282, a=1.406, b=2.163, c=1.21\end{array}$ & $\begin{array}{c}-0.55<\ln \sigma^{-1}< \\
1.31\end{array}$ & $0-30$ & $\begin{array}{l}\Lambda \text { CDM: } \\
\text { WMAP5 }\end{array}$ \\
\hline \begin{tabular}{|l|} 
Watson \\
et al. $(2013)$
\end{tabular} & $\begin{array}{l}f_{\mathrm{W}_{\mathrm{SO}}}(\sigma, z)=\Gamma(\Delta, \sigma, z) f_{\mathrm{T}}(\sigma, z), \\
(A, a, b, c)_{z=0}=(0.194,2.267,1.805,1.287), \\
(A, a, b, c)_{z>6}=(0.563,874,3.810,1.453), \\
(A, a, b, c)_{0<z<6}= \\
\Omega_{M}(z) \times\left(1.907(1+z)^{-3.216}+0.074,\right. \\
3.136(1+z)^{-3.058}+2.349, \\
\left.5.907 \times(1+z)^{-3.599}+2.344,1.318\right), \\
\Gamma(\Delta, \sigma, z)=C(\Delta)\left(\frac{\Delta}{178}\right)^{d(z)} \exp \left[\frac{p\left(1-\frac{\Delta}{178}\right)}{\sigma^{q}}\right], \\
C(\Delta)=0.947 \exp \left[0.023\left(\frac{\Delta}{178}-1\right)\right], \\
d(z)=-0.456 \Omega_{M}(z)-0.139, p=0.072, \\
q=2.130 .\end{array}$ & $\begin{array}{l}-0.55<\ln \sigma^{-1}< \\
1.05(z=0) \\
-0.06<\ln \sigma^{-1}< \\
1.024(z>0)\end{array}$ & $0-30$ & $\begin{array}{l}\Lambda \mathrm{CDM}: \\
\text { WMAP5 }\end{array}$ \\
\hline
\end{tabular}



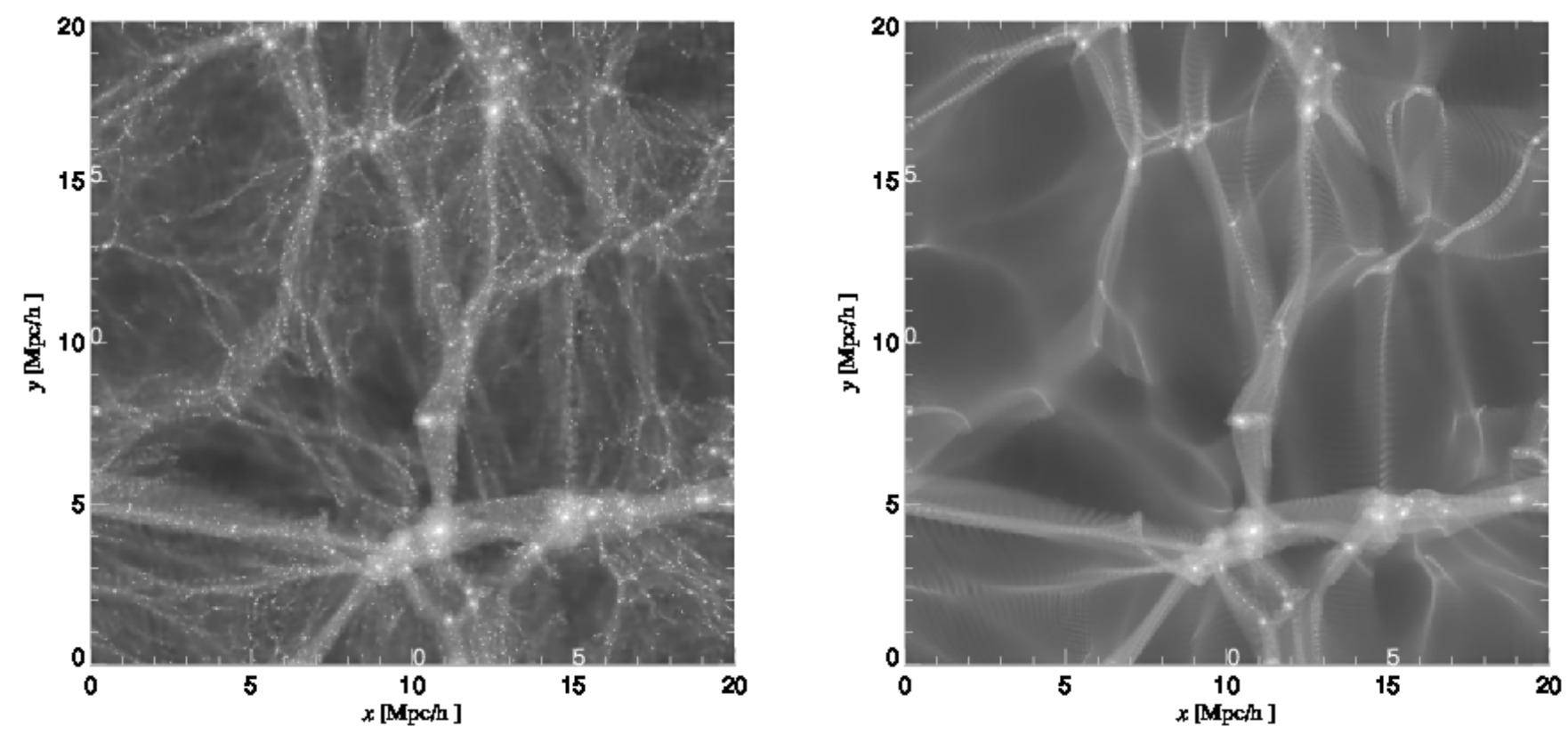

Figure 3: Visual impression of the projected dark matter density in a cosmological $N$-body simulations of a $20 h^{-1} \mathrm{Mpc}$ box, modelling the growth of structure in a fiducial CDM model (left panel) and its WDM counterpart (right panel). For the WDM model we assume a particle mass of $m_{X}=0.5 \mathrm{keV} / \mathrm{c}^{2}$. Note the absence of small-scale structure (i.e. low mass dark matter haloes) in the WDM run compared to the CDM run.

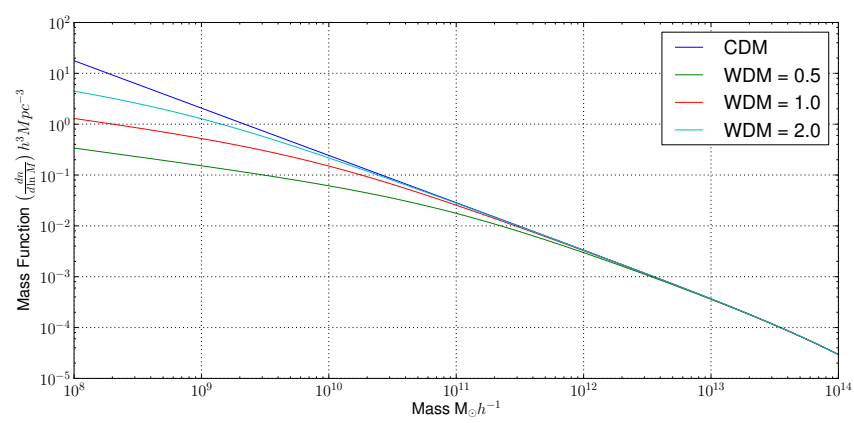

Figure 4: The HMF calculated assuming a fiducual CDM model and three different WDM models with particle masses of $m_{X}=0.5,1$ and $2 \mathrm{keV} / c^{2}$. Note the suppression of the number density of lower mass haloes.

1 and $2 \mathrm{keV} / \mathrm{c}^{2}-$ as the WDM particle mass decreases, so too does the number density of lower mass haloes, as we would expect. However, as we note in $\S 5$, there have been recent developments in the modelling of Warm Dark Matter models (e.g. Schneider et al., 2013, Pacucci et al. 2013 Benson et al. 2013) that we will incorporate in future versions of HMFcalc.

\section{Implementation}

HMFcalc has been designed to be visually intuitive, easy to learn and use, flexible and extensibility. These requirements guided our implementation;
- The code is written in Python, an extremely versatile language that is widely used in the astronomical community.

- The user interface is a web-app frontend, which is designed to work across multiple platforms and for its ease of use.

- The web-framework is provided by Django. It is flexible, easy to use and being written in Python, it integrates easily with the $\mathrm{hmf}$ backend.

- The whole code has been made open-source at https: //github.com/steven-murray/HMF. This way the code can be examined and updated, and also understood by those wishing to learn about the HMF.

We have also chosen to take an object-oriented approach, which makes sense because many of the HMF calculations share attributes. Class structures ${ }^{3}$ provide a way to define common attributes and make them available throughout all methods of the class, which in our case enables faster updating of parameters.

In the following subsections we describe in detail the backend $\mathrm{hmf}(\S 3.1$, before introducing the web-application (hereafter web-app) frontend HMFcalc.

\footnotetext{
${ }^{3}$ Objects to which attributes and methods may be attached.
} 


\subsection{HMFcalc's engine, hmf}

The calculation of the HMF is performed using $\mathrm{hmf}$, which consists of three python modules;

1. tools.py contains functions necessary for e.g. importing or generating (as required) the transfer function, calculation of the mass variance, interpolating the transfer function etc...;

2. cosmography.py contains functions that calculate cosmological distances and other cosmographic quantities;

3. hmf .py contains a single class dedicated to computing the HMF, as well as quantities such as the power spectrum, the mass variance, etc...

The handling of the transfer function in tools.py is done in one of two ways. If a filename is provided, the relevant file will be read and used as the transfer function. This file must be either produced by CAMB or in CAMB format. If no filename is provided, CAMB is called via pycamb ${ }^{4}$ an open-source python wrapper for CAMB. This enables efficient shared-memory multi-core calculation of the transfer function for a range of input parameters. The tools.py module also contains a function that checks the bounds on $k R$, as discussed in $\$ 2$.

$\mathrm{hmf}$. py is the central engine of $\mathrm{hmf}$, containing a single class named Perturbations that contains all attributes needed to calculate the HMF (the power spectrum, limits in $k$, the redshift, cosmology and mass variance etc.) and all the methods needed to calculate these quantities.

The constructor for the Perturbations class initialises an 'empty' object with a range of cosmological and numerical parameters set. The various quantities associated with the object are calculated only when necessary, by using the Qproperty decorator that allows the getting, setting and deleting of class properties in a flexible manner. These include the power spectrum (power), mass variance (sigma), growth factor (growth), effective spectral index (n_eff), fitting function (fsigma) and most importantly the differential and cumulative mass functions (dndlnm and ngtm).

Each fitting function is defined as a single method within the Perturbations class. However, the dndlnm method chooses between them using a dictionary indexed by string identifiers (such as 'ST' for Sheth et al. (2001) or 'Reed03' for Reed et al. (2003) ). Extending the range of fitting functions is as straightforward as writing a method containing the function definition, and registering it in the dictionary with a unique string identifier. However, users also have an option to pass a function in the format of a simple string that the class will parse and use as the fitting function.

Algorithm 11 summarises the steps necessary for the calculation of the differential HMF in hmf. Interpolation of the transfer function is performed with linear splines on the function in log-space, in which the function is almost perfectly linear over much of its range. This is consequently re-gridded between the given $k$-bounds in 4097

\footnotetext{
${ }^{4}$ https://github.com/joezuntz/pycamb
}

steps, to ensure sufficient accuracy for for romberg integration. Calculation of the cumulative HMF, $n(>M)$, is likewise performed using linear spline interpolation and extrapolation up to $M=10^{18} \mathrm{M}_{\odot} h^{-1}$ and consequent romberg integration using 4097 steps defined for each mass.

Users may want to re-calculate the HMF for different cosmological parameters, redshifts or fitting functions. To optimise this process, hmf tracks dependencies of quantities through chaining immediate links. For example, algorithm 1 demonstrates that the unnormalised power spectrum at the current epoch is dependent on the transfer function (and therefore all cosmological and numerical parameters involved in that calculation), and also the spectral index, but it does not depend on redshift, normalisation, or overdensity (either virial or critical). Any variable can be updated by calling the update() method with relevant keyword arguments; quantities that depend directly on the updated variables are deleted, which triggers deletion of quantities that depend directly on these and so forth. The next time any of these quantities are required, they are automatically re-calculated, using the updated variable. This system ensures that only the quantities that need to be re-calculated are re-calculated.

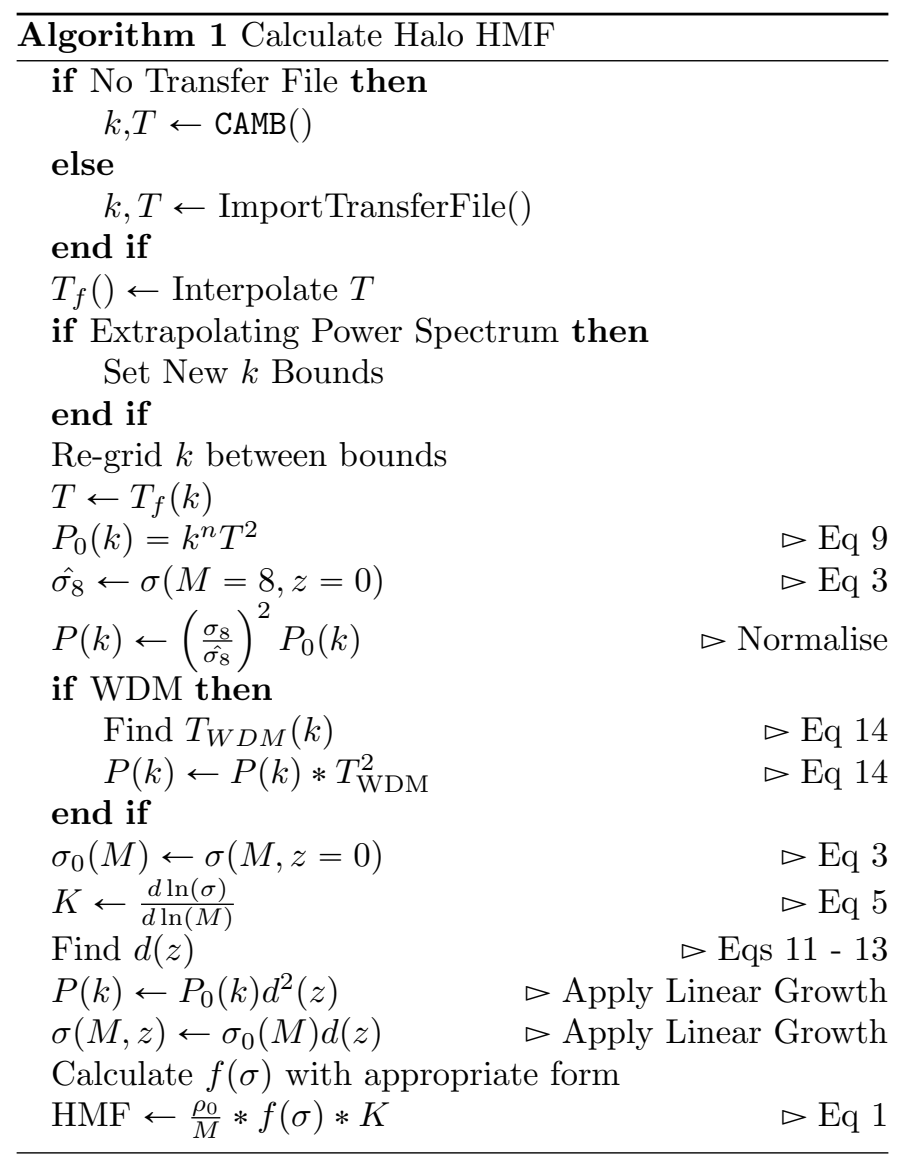

This updating method allows for the optimal calculation of many HMFs for varying parameters in an efficient and error-free manner. We have used it in Murray et al. (2013), generating 20,000 varying HMF realisations 
for each of 11 different fitting functions. On average the cascading method reduces processing time by $50 \%$ in tests where each available parameter is changed one time, although the size of reduction depends on which parameters are most often modified (e.g. changing only redshift reduces computations on average by more than $94 \%$ ).

\subsection{HMFcalc's web interface and the Django Framework}

Django provides a web-framework tuned to fast development and performance. Although developed with online newsrooms in mind, Django is easily adapted to scientific applications. A scientific Django web-app will generally be built around four parts - urls, views, templates and calculation - and Fig 3.2 depicts the flow of information within this framework 5 . The user initiates the information flow by pointing their browser at a particular URL. This URL is transformed to an address and contacts the Apache server on which HMCcalc is operating. The server translates the URL for input to the app's own urls.py module, which parses it and uses the information to choose a view function from the views.py module. This function may also be passed extra arguments from the input URL, based on query strings. The view chosen does all the logic, and dictates what should be seen on the final page. In the case of HMFcalc, one of the views accesses a form within the forms.py module for display. This form is passed through to a template, which is an enhanced html file. The template controls the presentation logic, putting the form fields down in appropriate positions on the page (with the help of CSS). The final template is then passed through to the server to be displayed by the browser.

We utilise a standard approach to the Django implementation. Nevertheless, we note some aspects of the design. Most of the views are defined as generic class-based views. This enables simpler construction and more flexibility in function. It also supports the creation of dynamic tabs for the user's navigation, via the plugin djangotabination. This implementation of the views is most important in the view that displays the main input form.

The view that displays the form is non-standard because there are two variants of it - one is for the initial creation of data, the other is to add more data. The forms for these differ in that the first lets the user choose a mass range (and grid), while the second does not - it uses the mass range specified previously. To create this functionality in the simplest way, we define a base form view, which implements the same logic for each form (i.e. what to do with the data after it has been successfully entered). We then create two subclasses, one for creation and one for addition of data. Each of these passes different arguments to the form class, which dynamically adds or removes the mass range inputs. This construction also allows for each

\footnotetext{
${ }^{5}$ Note that the 'Calculation Module' node is a black-box for the actual calculations using the hmf backend, which has been described already.
}

of these subclasses to define their own tab for user navigation.

Upon successful submission of the form, the base form view performs the main analysis. To do this, it takes the parsed input arguments and passes them to an external function that wraps around the hmf backend. For each combination of the input parameters, an HMF is produced and saved as an entry in a python dictionary with a key unique to the combination of parameters. This process is greatly simplified by using the update() method of the hmf package.

To enable later retrieval of the data for plotting and/or addition of extra data, the input parameters and calculated HMFs are saved to the session object, which runs continuously throughout the course of a user's session. It can be accessed by any view and provides a convenient way to transfer data. This is used as sparingly as possible, however, because it increases overall memory usage.

In terms of presentation, we utilise Django's templating system, combined with the bootstran ${ }^{6}$ CSS framework. This system allows for template inheritance - base templates can be written that define certain blocks that subtemplates may alter. This is convenient because we define a single base template for the whole site, which provides the general layout, including the navigation bar. The template for each page needs only to specify the unique content, which simplifies the design greatly. We use the bootstrap framework as it provides a simple means to create an aesthetically pleasing design.

Output and presentation of the calculations is done by means of the creation of figures and generation of downloadable ascii data. For both of these options, all files and resources are served in memory - nothing is written to disk on the server. This enhances performance and increases simplicity server-side. We have chosen to use matplotlib (Hunter, 2007), the standard scientific plotting library of Python, to drive the plotting for the application. This is used to produce PNG images for dynamic display on the web, and PDF images for optional download. Currently the plotting process is quite static - the data itself is the only dynamic aspect. This may result in plots that are not drawn in the best possible way for a given set of parameters. However, it is expected that those who wish to plot many different combinations will likely download the data file and come to their own plotting solution.

\subsection{Usage}

Using HMFcalc is straightforward - generation of a HMF is as easy as pressing "Calculate!" because the web form is populated with default parameters. Submission of the form generates a page with a plot of the calculated HMF with relevant legend, as well as a drop-down menu for choosing the particular additional functions to be displayed. These include the mass variance, the fitting

$6 \longdiv { \text { http://twitter.github.io/bootstrap/ } }$ 


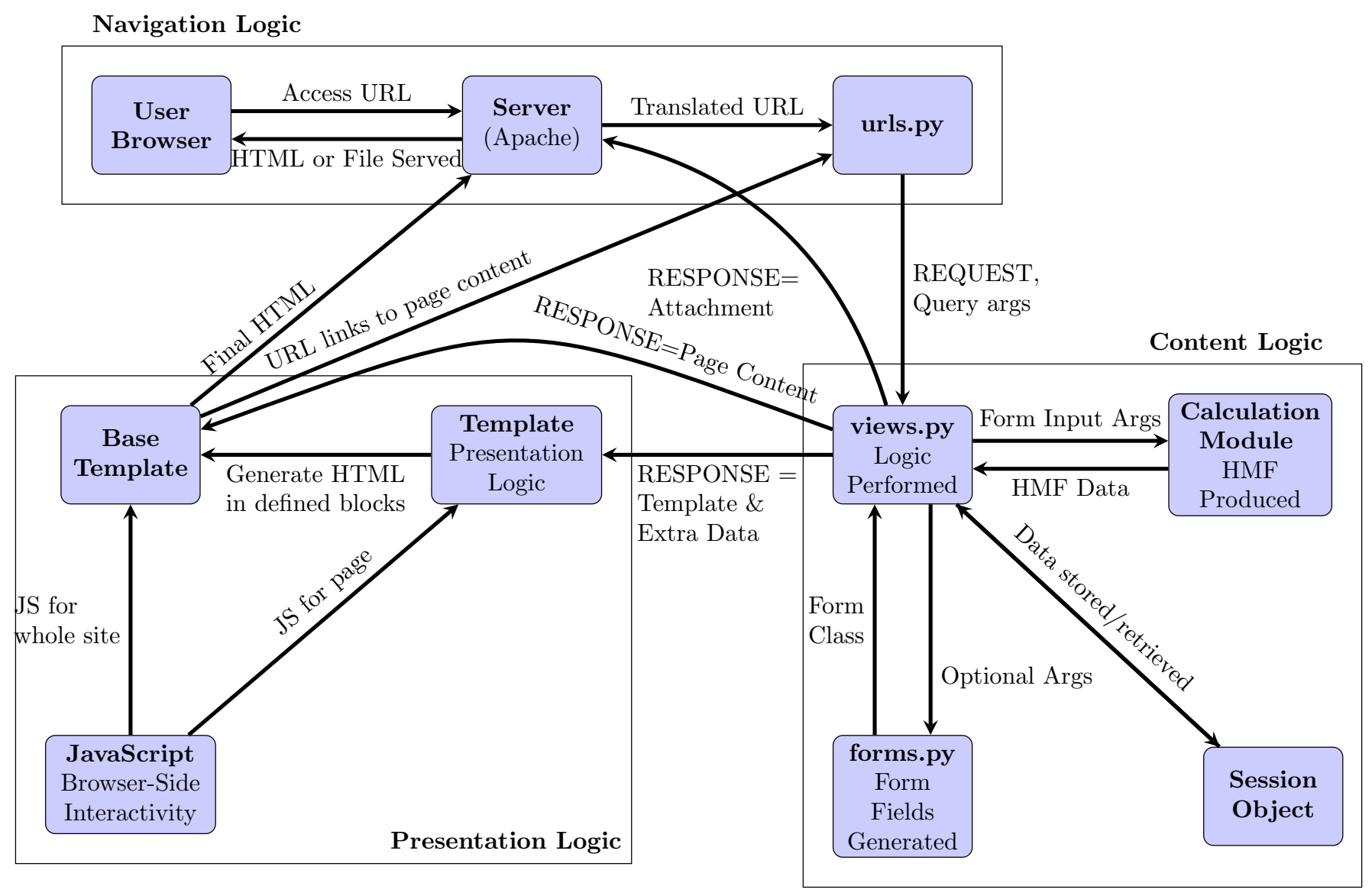

Figure 1: Flowchart depicting the several components of this django application and how they interact 
functions, the cumulative HMF and the power spectrum. Furthermore, an additional drop-down menu allows the user to download the currently viewed plot, all calculated plots, or data files from the calculation. Below the plot additional cosmographic quantities that have been calculated are presented (e.g. age of Universe at redshift $z$, distances, growth factors, etc...). In the following subsections we provide a glossary of parameters used in HMFcalc.

\subsubsection{Parameters}

The first category of parameters are the 'run parameters', which control the calculation (cf. Table 2). Where multiple parameters are possible, all combinations of parameters will be used in the calculation. This means that the number of HMFs generated can rise rapidly and so we place a limit on the total number of possible combinations.

The second category of parameters are the 'cosmological parameters' (cf. Table 3). The key parameter is the transfer function, which is invoked in one of three modes;

1. Pre-calculated (default); there are several transfer functions pre-calculated for commonly used cosmologies, each produced at high resolution by CAMB.

2. Custom; the user may upload their own transfer function, assumed to have been produced either using CAMB or in CAMB format.

3. On-the-fly; CAMB is run at invocation to produce the required transfer function.

If a pre-calculated transfer function is chosen, all cosmological parameters are set as read-only on the form; if a custom transfer function is used, the user must ensure that the cosmological parameters entered in the web-app match those used to generate the transfer function.

In Table 3, we use $\mathrm{T}, \mathrm{H}$ and $\mathrm{B}$ to indicate whether or not a parameter affects the transfer function $(\mathrm{T})$, the HMF $(\mathrm{H})$ or both $(\mathrm{B})$. Note that those that affect both need special care if the user uploads their own transfer function.

\subsubsection{An Important Caveat}

HMFcalc will calculate a HMF given any set of cosmological parameters and fitting function. However, most HMFs are empirical fits to the results of cosmological $N$ body simulations and are tied to specific cosmologies (but see, e.g. Jenkins et al., 2001, Courtin et al., 2010)); whether or not the HMF is universal remains a topic of active research (see discussion in Tinker and Kravtsov 2008, Bhattacharya et al. 2011 and Murray et al.|2013). For this reason we caution the careful user to compare the results of HMFcalc against N-body simulations of the appropriate cosmology.

\subsection{Comparison To Other Codes}

A small number of public codes are available for calculating the HMF - eg. genmf ${ }^{7}, \mathrm{MF}_{-} \mathrm{Code}^{8}$, and the HMF calculator in the yt project 9 . We have tested $\mathrm{hmf}$ against these codes and find excellent consistency. However, we note that HMFcalc has a number of advantages over these codes; it is inherently visually interactive and cross-platform, it allows for dynamic accurate calculation of the transfer function with CAMB and efficient and self-consistent parameter updates, and it can be easily extended by the user with bespoke fitting functions.

\section{Example Applications}

We have designed HMFcalc to be a useful tool for observers and theorists alike, and we expect the primary use of HMFcalc to be the quick and straightforward generation of HMFs for different fitting functions and as a function of cosmological parameters and cosmic epoch. However, HMFcalc should be a particularly useful aid to those who work with cosmological simulations, and we demonstrate how with two examples.

\subsection{Box Size for One Halo of Mass M}

A cosmological simulation follows the growth of structure in a periodic cubic box of volume $L^{3} \mathrm{Mpc}^{3} h^{-3}$ containing $N_{p}$ particles. In principle $L$ can be arbitrarily large, but normally it's set by requiring that the smallest resolved haloes contain a minimum number of particles, or equivalently the particle mass $m_{p} \propto L^{3} / N_{p}$. Given this restriction on $L$ enforced by $m_{p}$, it is interesting to ask how large the periodic box needs to be to contain at least one halo of mass $>M$. We require

$$
n(>M) L^{3} \geqslant 1 \rightarrow L \geqslant n(>M)^{-1 / 3} .
$$

We have chosen to create a method in Perturbations() (from the hmf backend) to derive Eq 16. We add the following code to the class:

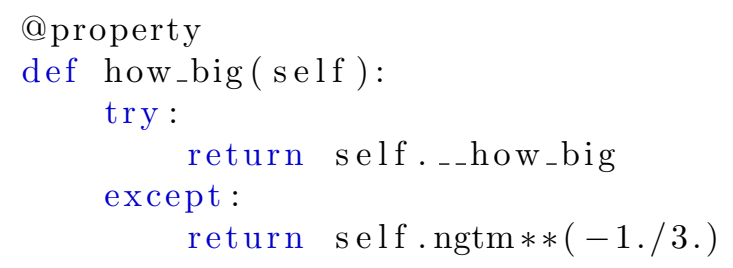

@how_big.deleter

def how_big( self):

try :

del self___how_big

except :

pass

7 http://icc.dur.ac.uk/Research/PublicDownloads/genmf_v1. 01.tar

http://cosmo.nyu.edu/ tinker/massfunction/MF_code.tar

http://yt-project.org/ 
Table 2: Description of Run Parameters

\begin{tabular}{|c|c|c|}
\hline PARAMETER & Mult? & DESCRIPTION \\
\hline Redshift & $\checkmark$ & $\begin{array}{l}\text { Epoch at which the HMF is calculated; default is } z=0 \text {. Affects HMF through } \\
\text { growth parameter (cf. } 22.2 \text {, quick to update. Some recent fitting functions contain } \\
\text { explicit redshift dependence. }\end{array}$ \\
\hline $\begin{array}{l}\text { Virial Overdensity } \\
\Delta_{\text {vir }}\end{array}$ & $\checkmark$ & $\begin{array}{l}\text { Defines the extent of a halo; default is } 178 \text {. Assumes haloes correspond to spherical } \\
\text { overdensities of } \Delta_{\text {vir }} \text { times the critical density at that epoch. Note Tinker and } \\
\text { Kravtsov (2008) and Watson et al. (2013) HMFs depend explicitly on } \Delta_{\text {vir }} \text {. }\end{array}$ \\
\hline WDM Mass $m_{X}$ & $\checkmark$ & $\begin{array}{l}\text { WDM particle mass in single-species models, in units of } \mathrm{keV} / \mathrm{c}^{2} \text {; default is } 0 \text {. This } \\
\text { is used to calculate the WDM transfer function and consequently the power } \\
\text { spectrum (cf. Eq 14). }\end{array}$ \\
\hline Fitting Function & $\checkmark$ & Defines fraction of mass collapsed at a given mass scale. See 2.1 for more details. \\
\hline $\begin{array}{l}\text { Custom Fitting } \\
\text { Function }\end{array}$ & $\boldsymbol{x}$ & $\begin{array}{l}\text { User-defined fitting function, written in Python syntax as a string, with } \\
\text { independent variable named } x \text { corresponding to the mass variance; default is } \\
\text { empty. To add more complex functions, the user will need to edit the source code } \\
\text { itself. }\end{array}$ \\
\hline Extrapolate? & $\boldsymbol{x}$ & $\begin{array}{l}\text { Enables/disables extrapolation the transfer function in wavenumber } k \text { beyond } \\
\text { tabulated bounds; default is enabled. Assumes transfer function is linear (in log } \\
\text { space) near bounds and so can be easily extrapolated. }\end{array}$ \\
\hline $\operatorname{Min} \& \operatorname{Max} k$ & $\checkmark$ & $\begin{array}{l}\text { Minimum and maximum wavenumber }(k) \text { for the power spectrum; defaults are } \\
\sim 10^{-8} \text { and } 2000 \text { in units of } h \mathrm{Mpc}^{-1} \text { for accurate prediction }(\mathrm{cf.} .2)\end{array}$ \\
\hline Min \& Max Mass & $x$ & $\begin{array}{l}\text { Defines minimum and maximum halo mass bounds for plotted HMF; defaults are } 8 \\
\text { and } 15 \text { in units of } \log _{10} h^{-1} \mathrm{M}_{\odot} \text {. }\end{array}$ \\
\hline Mass Bin Width & $\boldsymbol{*}$ & Defines logarithmic mass bin width; default is 0.05 dex. \\
\hline
\end{tabular}

Table 3: Description of Cosmological Parameters

\begin{tabular}{|c|c|c|}
\hline PARAMEter & AfFECTS & DESCRIPTION \\
\hline Transfer Function & - & $\begin{array}{l}\text { Choose from drop-down menu. Either pre-calculated and based on popular } \\
\text { cosmologies or customised based on the available parameters. }\end{array}$ \\
\hline HMF Labels & - & $\begin{array}{l}\text { Must be unique - one for each parameter set plotted. Appears in plots as legends } \\
\text { and in downloadable data as column headers. }\end{array}$ \\
\hline$\delta_{c}$ & $\mathrm{H}$ & $\begin{array}{l}\text { Critical overdensity for collapse. Although cosmology-dependent, we assume the } \\
\text { Einstein-de Sitter value of } 1.686 \text { as default. }\end{array}$ \\
\hline$n_{s}$ & $\mathrm{H}$ & Spectral index of power spectrum - see $\$ 2$. \\
\hline$\sigma_{8}$ & $\mathrm{H}$ & $\begin{array}{l}\text { Mass variance on scale of } 8 h^{-1} \mathrm{Mpc} \text { at } z=0 \text { used to normalise power spectrum - } \\
\text { (see } \S 2 \text {. }\end{array}$ \\
\hline$H_{0}$ & $\mathrm{~T}$ & Hubble Parameter at $z=0$. \\
\hline$\Omega_{b}$ & $\mathrm{~B}$ & Baryon density at $z=0$. \\
\hline$\Omega_{c}$ & B & Dark matter density at $z=0$. \\
\hline$\Omega_{\Lambda}$ & B & Dark energy density at $z=0$. \\
\hline
\end{tabular}


This is all that is needed to use hmf to calculate Eq 16 . Note the eproperty decorator ensures that the value of how_big will change as the value of ngtm is modified. We extend the web interface to output this information by adding the following to the function $\mathrm{hmf}$ _driver () in utils.py towards the end of the loops;

if 'get_L' in extra_plots :

$$
\begin{gathered}
\text { mass_data }[" \mathrm{~L}(\mathrm{~N}=1) \text { _" }+\operatorname{getname}(\text { labels })]= \\
\text { pert.how_big }
\end{gathered}
$$

Here, the extra_plots variable is a list that contains the names of the extra plots to be calculated (defined in the form later). The function is saved to the existing mass_data array, under a label beginning with L $(\mathrm{N}=1)_{-}$, which describes the "box size needed for one halo". The Perturbations() object has been previously initialized as pert, and thus all we must do is access its how_big property.

As we have noted, we must implement the choice of calculating this function on the form. To do this we add the entry

$$
\text { ('get_L', 'Box Size for One Halo'), }
$$

to the optional_plots list variable in the HMFInput() class in forms.py. Its information will be passed to the form template, which will render it automatically in the 'Optional Extra Plots' fieldset.

We also need to create a conditional entry for the function in the drop-down menu of the results page, so that we can view the plot. To do this we add the following code to the __init__() method of the PlotChoices() class of forms.py:

$$
\begin{aligned}
& \text { if 'get_L' in session_plots: } \\
& \text { extra_plots.append (('L', } \\
& \text { 'Box Size for One Halo')) }
\end{aligned}
$$

Here we add the choice only if get_L was checked on the initial input form. The first argument is the name of the choice internally, while the second is the label that the user sees.

The only remaining task is to create the plot definition for the function, in views.py. We add the following code to produce the required axis labels and extract the $L(N=$ 1) function from the whole dataset:

$$
\begin{aligned}
& \text { elif plottype }=\text { 'L': } \\
& \text { keep }=[\text { string for string in mass_data } \\
& \text { if string. startswith ( } \left.\left.\left.\mathrm{L}(\mathrm{N}=1)_{-}\right)^{\prime}\right)\right] \\
& \text { title="Box Size, L, for One Halo" } \\
& \text { ylab="Box Size, L (Mpc/h)" } \\
& \text { yscale }=\text { 'log' }
\end{aligned}
$$

and add the plottype to the first list, mass_plots, specifying that the $x$-axis will be based on the mass grid. Here the plottype refers to the internal label from the PlotChoice() form. The first line chooses all the columns whose labels begin with the specified string, corresponding to our custom function. Note that these code samples

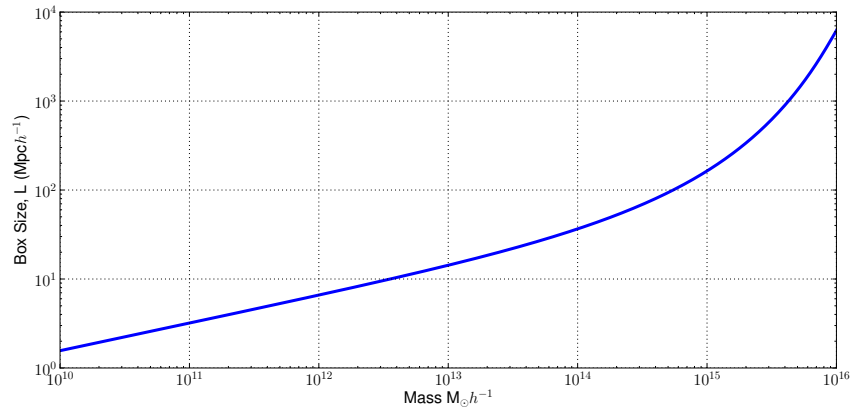

Figure 5: How large a box $L$ is required to form at least one halo of mass $>M$ ? This shows the result for a WMAP7 cosmology at $z=0$.

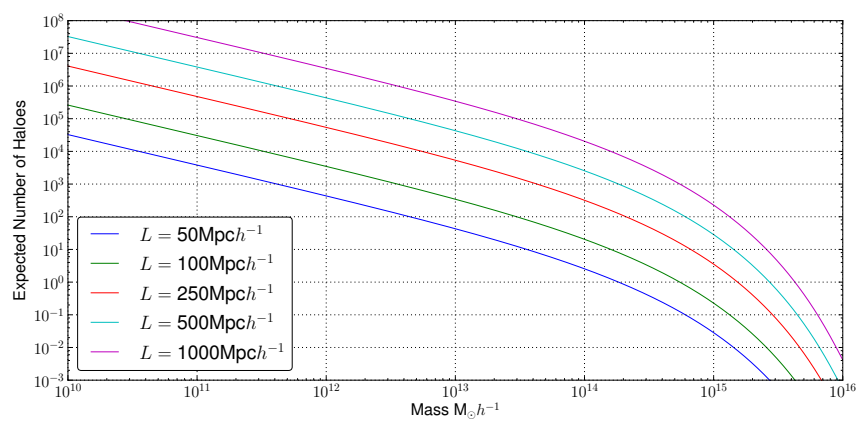

Figure 6: How many haloes do we expect of mass $>M$ in a simulation box of size $L$ ? This shows the result for a WMAP7 cosmology at $z=0$.

are merely indicative, nevertheless, the pattern will remain conceptually the same in future versions.

The result, for the WMAP7 cosmology at $z=0$, is shown in Fig 5 , it is computed by default by the web-app. We have also determined how many haloes are expected in a simulation of box size $L$ (see Fig 6). This is not currently implemented in the web-app, but it will be in future versions.

\subsection{The Impact of Finite Box Size}

Cosmological simulations model the growth of structure over cosmic time by following the evolution of matter density perturbations in a periodic box. Because the box has a finite size $L$, the longest wavelength perturbation that can be reliably modeled will have a wavenumber $k_{\text {min }}$ of

$$
k_{\min }=\frac{2 \pi}{L} .
$$

This means that the effect of perturbations with wavelengths longer than the box size cannot be captured, and implies that the mass variance we would expect (cf. Eq3) will be larger than the one that we measure. This effect, and its influence on the HMF at higher masses, has been noted in previous studies (e.g Bagla and Ray, 2005; Power and Knebe, 2006; Lukić et al., 2007).

We can use HMFcalc to quantify this effect for boxes of size 50, 100, 250 and $500 h^{-1} \mathrm{Mpc}$, corresponding to 


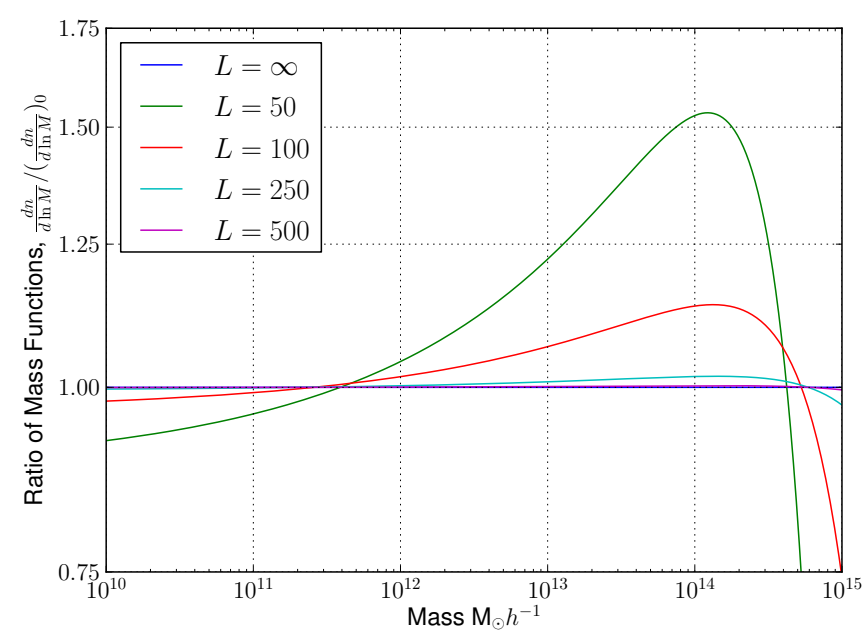

Figure 7: Artificial enhancement of the number density of haloes of mass $\sim 10^{14} \mathrm{M}_{\odot} h^{-1}$ in smaller boxes.

$k_{\text {min }}=0.1257,0.0628,0.02513$ and $0.01257 \mathrm{hMpc}^{-1}$ in Eq 17. We use the inbuilt functionality of HMFcalc to enter as input all of these minimum $k$ bounds at once, plus a baseline of $k_{\min }=10^{-8}$ that corresponds to the limit of infinite box size. The result is shown in figure 4.2, which shows that the effect of box size is most severe for the $50 h^{-1} \mathrm{Mpc}$, with the number density of $10^{1} 4 \mathrm{M}_{\odot} h^{-1}$ mass haloes enhanced by $\sim 50 \%$; is weaker for the $100 h^{-1} \mathrm{Mpc}$ box; and is negligible for box sizes as large as $250 h^{-1} \mathrm{Mpc}$. These results are in good agreement with the findings of Power and Knebe (2006).

\section{Future Development}

\subsection{Extending Range of Dark Matter and Dark Energy Models}

In the current version of HMFcalc, we have considered only the fiducial Cold Dark Matter model and a straightforward extension to model Warm Dark Matter, using the framework set out in (Bode et al. 2001). In forthcoming versions of HMFcalc, we shall update the framework to account for more recent developments in the modelling of Warm Dark Matter models (e.g. Schneider et al., 2013, Pacucci et al., 2013, Benson et al., 2013), as well as alternative dark energy models such as those explored in, for example, Francis et al. (2009) and Courtin et al. (2010).

\subsection{Calibrating Synthetic Galaxy Surveys}

"Halo Occupation Distribution" (HOD) models parameterise how likely galaxies are to occupy dark matter haloes and provide a convenient framework for creating synthetic galaxy surveys. Formally, the HOD defines a probability $P(N \mid M)$ of finding $N$ luminous galaxies in a halo of mass $M$ (Berlind and Weinberg, 2002), and it allows a population of dark matter haloes to be mapped to a synthetic galaxy population whose abundance and spatial clustering can be tuned to reproduce the observed galaxy population. We will extend HMFcalc so that we can take as input a given set of cosmological parameters, a dark matter and dark energy model, and a set of observational constraints (e.g. 2-point correlation function in different wavelength bands) and calibrate HOD parameters to reproduce the properties of the observed galaxy population. With this framework we will develop empirically-motivated synthetic galaxy surveys using both the outputs from both cosmological $N$-body simulations (e.g. Springel et al., 2006) as well as faster, more approximate schemes such as $2^{\text {nd }}$ order Lagrangian perturbation theory (e.g. Manera et al., 2013), vital in the coming era of large galaxy surveys.

\subsection{Dynamic and Adaptable User Interface}

We are developing HMFcalc into a dynamic and adaptable online tool that will give the user finer and more varied control over the generation of data. For example, we would like users to be able to explore how variations in the cosmological parameters influence the HMF and to see the resulting HMF update in real-time. A similar framework will be used to allow users to explore how HOD-derived galaxy population observables vary with changes in cosmological parameters and dark matter/dark energy parameters. We would also like users to be able to explore how, for example, changing from the traditional top-hat window function (cf. Eq 4 to sharp- $k$ filter window functions (cf. Schneider et al., 2013) influence the predicted HMF, especially in WDM models. Such an online toolbox will provide an invaluable community resource for both observers and theorists alike.

\section{Summary}

We have presented HMFcalc, a flexible, efficient and easy to use web application for calculating the dark matter halo mass function (HMF), a fundamental property of cosmological structure formation models. HMFcalc and its engine hmf are implemented in Python, which offers a simple yet powerful and extensible cross-platform method for building community software. Observers and theorists alike should find HMFcalc a valuable resource to rapidly explore the effects of cosmological parameters, redshift and fitting function on the predicted HMF, while cosmological simulators will find it a useful tool for understanding, for example, the biases introduced by finite simulation volume. In future work we will extend HMFcalc to give the user greater flexibility in how they interact with their data; we will broaden the range of dark matter and dark energy models considered; and finally, we will use the framework provided by HMFcalc to develop the infrastructure for fast approximate methods for generating bespoke synthetic galaxy surveys. 


\section{Acknowledgments}

ASGR acknowledges support of a UWA postdoctoral research fellowship. Part of this research was undertaken as part of the Survey Simulation Pipeline (SSimPL; ssimpluniverse.tk). The Centre for All-Sky Astrophysics (CAASTRO) is an Australian Research Council Centre of Excellence, funded by grant CE11E0090.

\section{References}

Ade, P. A. R., Aghanim, N., Armitage-Caplan, C., Arnaud, M., Ashdown, M., Atrio-Barandela, F., Aumont, J., Baccigalupi, C., Banday, A. J., et al., Mar. 2013. Planck 2013 results. XVI. Cosmological parameters.

Angulo, R. E., Springel, V., White, S. D. M., Jenkins, A., Baugh, C. M., Frenk, C. S., Nov. 2012. Scaling relations for galaxy clusters in the Millennium-XXL simulation. MNRAS 426, 2046-2062.

Bagla, J. S., Ray, S., Apr. 2005. Comments on the size of the simulation box in cosmological N-body simulations. MNRAS 358, 10761082 .

Benson, A. J., Farahi, A., Cole, S., Moustakas, L. A., Jenkins, A., Lovell, M., Kennedy, R., Helly, J., Frenk, C., Jan. 2013. Dark matter halo merger histories beyond cold dark matter - I. Methods and application to warm dark matter. MNRAS 428, 1774-1789.

Bergström, L., May 2000. Non-baryonic dark matter: observational evidence and detection methods. Reports on Progress in Physics 63 (5), 793-841.

Berlind, A. A., Weinberg, D. H., Aug. 2002. The Halo Occupation Distribution: Toward an Empirical Determination of the Relation between Galaxies and Mass. The Astrophysical Journal 575 (2), $587-616$.

Bhattacharya, S., Heitmann, K., White, M., Lukić, Z., Wagner, C., Habib, S., May 2011. MASS FUNCTION PREDICTIONS BEYOND $\Lambda$ CDM. The Astrophysical Journal 732 (2), 122.

Bode, P., Ostriker, J. P., Turok, N., Jul. 2001. Halo Formation in Warm Dark Matter Models. The Astrophysical Journal 556 (1), 93-107.

Bond, J. R., Cole, S., Efstathiou, G. P., Kaiser, N., Oct. 1991. Excursion set mass functions for hierarchical Gaussian fluctuations. The Astrophysical Journal 379, 440.

Courtin, J., Rasera, Y., Alimi, J.-M., Corasaniti, P. S., Boucher, V., Füzfa, a., Oct. 2010. Imprints of dark energy on cosmic structure formation - II. Non-universality of the halo mass function. Monthly Notices of the Royal Astronomical Society 1931, no-no.

Crocce, M., Fosalba, P., Castander, F. J., Gaztañaga, E., Apr. 2010. Simulating the Universe with MICE: the abundance of massive clusters. Monthly Notices of the Royal Astronomical Society 403 (3), 1353-1367.

Driver, S. P. e. a., May 2011. Galaxy and Mass Assembly (GAMA): survey diagnostics and core data release. Monthly Notices of the Royal Astronomical Society 413 (2), 971-995.

Francis, M. J., Lewis, G. F., Linder, E. V., Feb. 2009. Halo mass functions in early dark energy cosmologies. Monthly Notices of the Royal Astronomical Society: Letters 393 (1), L31-L35.

Hunter, J. D., 2007. Matplotlib: A 2d graphics environment. Computing In Science \& Engineering 9 (3), 90-95.

Jenkins, A. R., Frenk, C. S., White, S. D. M., Colberg, J. M., Cole, S., Evrard, a. E., Couchman, H. M. P., Yoshida, N., Feb. 2001. The mass function of dark matter haloes. Monthly Notices of the Royal Astronomical Society 321 (2), 372-384.

Knebe, A., Pearce, F. R., Lux, H., Ascasibar, Y., Behroozi, P., Casado, J., Corbett Moran, C., Diemand, J., Dolag, K. Dominguez-Tenreiro, R., Elahi, P., Falck, B., Gottloeber, S., Han, J., Klypin, A., Lukic, Z., Maciejewski, M., McBride, C. K., Merchan, M. E., Muldrew, S. I., Neyrinck, M., Onions, J., Planelles, S., Potter, D., Quilis, V., Rasera, Y., Ricker, P. M., Roy, F., Ruiz, A. N., Sgro, M. A., Springel, V., Stadel, J., Sutter, P. M., Tweed,
D., Zemp, M., Apr. 2013. Structure Finding in Cosmological Simulations: The State of Affairs. ArXiv e-prints.

Lewis, A., Challinor, A., Lasenby, A., Aug. 2000. Efficient Computation of Cosmic Microwave Background Anisotropies in Closed FriedmannRobertsonWalker Models. The Astrophysical Journal 538 (2), 473-476.

Lukić, Z., Heitmann, K., Habib, S., Bashinsky, S., Ricker, P. M., Dec. 2007. The Halo Mass Function: HighRedshift Evolution and Universality. The Astrophysical Journal 671 (2), 1160-1181.

Manera, M., Scoccimarro, R., Percival, W. J., Samushia, L., McBride, C. K., Ross, A. J., Sheth, R. K., White, M., Reid, B. A., Sánchez, A. G., de Putter, R., Xu, X., Berlind, A. A., Brinkmann, J., Maraston, C., Nichol, B., Montesano, F., Padmanabhan, N., Skibba, R. A., Tojeiro, R., Weaver, B. A., Jan. 2013. The clustering of galaxies in the SDSS-III Baryon Oscillation Spectroscopic Survey: a large sample of mock galaxy catalogues. MNRAS 428, 1036-1054.

Murray, S., Power, C., Robotham, A., Jun. 2013. How well do we know the Halo Mass Function? ArXiv e-prints.

Pacucci, F., Mesinger, A., Haiman, Z., May 2013. Focusing on Warm Dark Matter with Lensed High-redshift Galaxies. ArXiv e-prints.

Pierre, M., Pacaud, F., Juin, J. B., Melin, J. B., Valageas, P., Clerc, N., Corasaniti, P. S., Jun. 2011. Precision cosmology with a wide area XMM cluster survey. MNRAS 414, 1732-1746.

Power, C., Knebe, A., Aug. 2006. The impact of box size on the properties of dark matter haloes in cosmological simulations. MNRAS 370, 691-701.

Press, W. H., Schechter, P., 1974. Formation of galaxies and clusters of galaxies by self-similar gravitational condensation. The Astrophysical Journal 187, 425-438.

Reed, D., Gardner, J., Quinn, T., Stadel, J., Fardal, M., Lake, G., Governato, F., Dec. 2003. Evolution of the mass function of dark matter haloes. Monthly Notices of the Royal Astronomical Society 346 (2), 565-572.

Reed, D. S., Bower, R., Frenk, C. S., Jenkins, A., Theuns, T., Jan. 2007. The halo mass function from the dark ages through the present day. Monthly Notices of the Royal Astronomical Society 374 (1), 2-15.

Schneider, A., Smith, R. E., Reed, D., Jun. 2013. Halo mass function and the free streaming scale. MNRAS.

Sheth, R. K., Mo, H. J., Tormen, G., May 2001. Ellipsoidal collapse and an improved model for the number and spatial distribution of dark matter haloes. Monthly Notices of the Royal Astronomical Society 323 (1), 1-12.

Springel, V., Frenk, C. S., White, S. D. M., Apr. 2006. The largescale structure of the Universe. Nature 440, 1137-1144.

Tinker, J., Kravtsov, A. V., 2008. Toward a halo mass function for precision cosmology: the limits of universality. The Astrophysical Journal 688, 709-728.

Vikhlinin, a., Kravtsov, a. V., Burenin, R. a., Ebeling, H., Forman, W. R., Hornstrup, a., Jones, C., Murray, S. S., Nagai, D., Quintana, H., Voevodkin, a., Feb. 2009. Chandra Cluster Cosmology Project Iii: Cosmological Parameter Constraints. The Astrophysical Journal 692 (2), 1060-1074.

Warren, M. S., Abazajian, K., Holz, D. E., Teodoro, L., Aug. 2006. Precision Determination of the Mass Function of Dark Matter Halos. The Astrophysical Journal 646 (2), 881-885.

Watson, W. A., Iliev, I. T., D'Aloisio, A., Knebe, A., Shapiro, P. R., Yepes, G., Jun. 2013. The halo mass function through the cosmic ages. MNRAS.

White, S. D. M., 2002. The Mass Function. The Astrophysical Journal Supplement Series 143, 241-255. 